\title{
CONGRUENCES IN ORDERED PAIRS OF PARTITIONS
}

\author{
PAUL HAMMOND and RICHARD LEWIS
}

Received 28 November 2003 and in revised form 12 December 2003

\begin{abstract}
Dyson defined the rank of a partition (as the first part minus the number of parts) whilst investigating certain congruences in the sequence $p_{-1}(n)$. The rank has been widely studied as have been other statistics, such as the crank. In this paper a "birank" is defined which relates to ordered pairs of partitions, and is used in an elementary proof of a congruence in $p_{-2}(n)$.
\end{abstract}

2000 Mathematics Subject Classification: 05A17, 11P81, 11 P83.

1. Introducing the birank. A partition is defined as being a nonincreasing sequence of positive integers, $\lambda=\left(\lambda_{1}, \lambda_{2}, \ldots, \lambda_{r}\right)$. The set of all partitions, which includes the empty partition $\varnothing$, is denoted by $\mathscr{P}$. The sum of the parts of a given partition is called the weight of the partition, $\operatorname{wt}(\lambda)=\lambda_{1}+\lambda_{2}+\cdots+\lambda_{r}$. It is standard notation to write $(z ; q)_{\infty}:=\prod_{t \geq 0}\left(1-z q^{t}\right)$ and $p_{-k}(n)$ for the coefficient of $q^{n}$ in $(q ; q)_{\infty}^{-k}$, for fixed $k$. It is easy to show that the number of partitions of weight $n$ is $p_{-1}(n)$ (for the empty partition, wt $(\lambda)=0$ ). It is also easy to show that the number of ordered pairs of partitions of weight $n$ is $p_{-2}(n)$, the weight of an ordered pair being defined as the sum of the weights of the two partitions in the pair. The sequence $p_{-1}(n)$ is known to satisfy certain congruences, one of which

$$
p_{-1}(5 n+4) \equiv 0 \bmod 5
$$

was noticed, and first proved, by Ramanujan (see [7]). In fact (1.1) is [6, Theorem 359], where an elementary proof is given. A similar, and equally elementary proof can be found for the following congruences:

$$
p_{-2}(5 n+2) \equiv p_{-2}(5 n+3) \equiv p_{-2}(5 n+4) \equiv 0 \bmod 5 \text {. }
$$

What would be desirable is a combinatorial reason why these congruences hold, a way in which, for $x=2,3,4$, the ordered pairs of weight $5 n+x$ could be split into five equinumerous sets.

With this in mind, we define the birank $b(\pi)$ of an ordered pair of partitions $\pi=$ $(\lambda(1), \lambda(2))$ as the number of parts in the first partition minus the number of parts in the second partition. Thus $b(\pi):=\#(\lambda(1))-\#(\lambda(2))$. The number of ordered pairs of partitions of weight $n$ having birank $m$ will be written as $R(m, n)$, and $R(r, m, n)$ is the number of such ordered pairs having birank congruent to $r$ modulo $m$.

Now, $b(\lambda(1), \lambda(2))=-b(\lambda(2), \lambda(1))$, hence $R(m, n)=R(-m, n)$ and so $R(r, m, n)=$ $R(m-r, m, n)$. Thus, the birank bears at least a passing resemblance to Dyson's rank, 
which he introduced as an explanation for the congruence (1.1), in the same way (it will be shown) the birank explains (1.2). Dyson stated his conjectures in [2] and they were proved in [1].

Now, summing over all ordered pairs of partitions gives

$$
\sum_{\pi \in \mathscr{P}^{2}} z^{b(\pi)} q^{\mathrm{wt}(\pi)}=\frac{1}{(z q ; q)_{\infty}\left(z^{-1} q ; q\right)_{\infty}} .
$$

Hence the generating function for $R(m, n)$ is

$$
\sum_{m \in \mathbb{Z}} \sum_{n \geq 0} R(m, n) z^{m} q^{n}=\frac{1}{(z q ; q)_{\infty}\left(z^{-1} q ; q\right)_{\infty}} .
$$

Thus, multiplying the generating function for the birank by $(q ; q)_{\infty}$ gives the generating function for the crank of Andrews and Garvan, see [3, identity (3.8)].

The rank, and later the crank, have been widely studied in connection with congruences, such as (1.1) in the sequence $p_{-1}(n)$. There is a relative shortage of published work on Dyson-type ranks related to congruences in sequences $p_{-k}(n)$ for $k>1$ (see Acknowledgment).

The following abbreviation will be used later:

$$
R[\cdot]:=\sum_{n \geq 0} R(\cdot, 5, n) q^{n}
$$

The only tool we use to investigate the behaviour of the birank is the triple product identity, a proof of which can be found in [6]. The identity states that if $[z ; q]:=$ $(z ; q)_{\infty}\left(z^{-1} q ; q\right)_{\infty}$, then

$$
[z ; q](q ; q)_{\infty}=\sum_{n \in \mathbb{Z}}(-1)^{n} z^{n} q^{n(n-1) / 2}=\sum_{n \geq 0}(-1)^{n}\left(z^{-n}-z^{n+1}\right) q^{n(n+1) / 2} .
$$

Dividing each side by $1-z$ gives

$$
(z q ; q)_{\infty}\left(z^{-1} q ; q\right)(q ; q)_{\infty}=\sum_{n \geq 0}(-1)^{n}\left(z^{n}+z^{n-1}+\cdots+z^{-n}\right) q^{n(n+1) / 2} .
$$

2. The behaviour of the birank. The congruence (1.2) is explained by the last three lines of the following theorem.

THEOREM 2.1.

$$
\begin{gathered}
R(0,5,5 n)>R(1,5,5 n)=R(2,5,5 n), \\
R(1,5,5 n+1)>^{*} R(0,5,5 n+1)=R(2,5,5 n+1), \\
R(0,5,5 n+2)=R(1,5,5 n+2)=R(2,5,5 n+2), \\
R(0,5,5 n+3)=R(1,5,5 n+3)=R(2,5,5 n+3), \\
R(0,5,5 n+4)=R(1,5,5 n+4)=R(2,5,5 n+4) .
\end{gathered}
$$

The only exception, $(*)$, is $R(0,5,6)=R(1,5,6)$. 
Proof. Putting $z=\omega$, where $\omega=e^{2 \pi i / 5}$ in (1.4) gives

$$
\sum_{m \in \mathbb{Z}} \sum_{n \geq 0} R(m, n) \omega^{m} q^{n}=\frac{1}{(\omega q ; q)_{\infty}\left(\omega^{-1} q ; q\right)_{\infty}}=\frac{\left(\omega^{2} q ; q\right)_{\infty}\left(\omega^{-2} q ; q\right)_{\infty}(q ; q)_{\infty}}{\left(q^{5} ; q^{5}\right)_{\infty}} .
$$

The term on the right is, by (1.7), equal to

$$
\frac{1}{\left(q^{5} ; q^{5}\right)_{\infty}} \sum_{n \geq 0}(-1)^{n}\left(\omega^{2 n}+\omega^{2 n-2}+\cdots+\omega^{-2 n}\right) q^{n(n+1) / 2}
$$

because $\omega^{2}+\omega+1+\omega^{-1}+\omega^{-2}=0$, this can be simplified and then dissected to give

$$
\frac{1}{\left(q^{5} ; q^{5}\right)_{\infty}}\left(\sum_{n \in \mathbb{Z}}(-1)^{n} q^{\left(25 n^{2}+5 n\right) / 2}+\left(\omega+\omega^{-1}\right) q \sum_{n \in \mathbb{Z}}(-1)^{n} q^{\left(25 n^{2}+15 n\right) / 2}\right),
$$

which, by (1.6), is equal to

$$
\frac{\left(q^{25} ; q^{25}\right)_{\infty}}{\left(q^{5} ; q^{5}\right)_{\infty}}\left(\left[q^{10} ; q^{25}\right]+\left(\omega+\omega^{-1}\right) q\left[q^{5} ; q^{25}\right]\right)=\frac{1}{\left[q^{5} ; q^{25}\right]}+\left(\omega+\omega^{-1}\right) \frac{q}{\left[q^{10} ; q^{25}\right]} .
$$

Recalling (1.5), it is clear that

$$
\sum_{m \in \mathbb{Z}} \sum_{n \geq 0} R(m, n) w^{m} q^{n}=R[0]+\omega R[1]+\omega^{2} R[2]+\omega^{3} R[3]+\omega^{4} R[4]
$$

Now, since $\omega^{2}=-1-\omega-\omega^{3}-\omega^{4}, R[1]=R[4]$, and $R[2]=R[3]$,

$$
R[0]-R[2]+\left(\omega+\omega^{4}\right)(R[1]-R[2])=\frac{1}{\left[q^{5} ; q^{25}\right]}+\left(\omega+\omega^{-1}\right) \frac{q}{\left[q^{10} ; q^{25}\right]} .
$$

From identity (2.7), which is [4, Lemma 3.9], and the irrationality of $\omega+\omega^{-1}$, it follows that

$$
\begin{aligned}
& \sum_{n \geq 0}[R(0,5, n)-R(2,5, n)] q^{n}=\frac{1}{\left[q^{5} ; q^{25}\right]} \\
& \sum_{n \geq 0}[R(1,5, n)-R(2,5, n)] q^{n}=\frac{q}{\left[q^{10} ; q^{25}\right]}
\end{aligned}
$$

Now if the coefficient of $q^{n}$ in the expression on the right-hand side of (2.8) is, say, $c(n)$ and the coefficient in the expression on the right-hand side of (2.9) is $d(n)$, then it is clear that $c(n) \geq 0$ and the inequality is strict precisely when $n \equiv 0 \bmod 5$. Likewise, $d(n) \geq 0$ and the inequality is strict precisely when $n \equiv 1 \bmod 5$ (with one exception, $d(6)=0)$. This proves (2.1).

ACKNOWLEDGMENT. We are grateful to the anonymous referee for drawing our attention to the existence of a rank related to $p_{-24}(n)$ which is introduced in [5]. 


\section{REFERENCES}

[1] A. O. L. Atkin and P. Swinnerton-Dyer, Some properties of partitions, Proc. London Math. Soc. (3) 4 (1954), 84-106.

[2] F. J. Dyson, Some guesses in the theory of partitions, Eureka 8 (1944), 10-15.

[3] F. G. Garvan, Combinatorial interpretations of Ramanujan's partition congruences, Ramanujan Revisited (Urbana-Champaign, Ill, 1987), Academic Press, Massachusetts, 1988, pp. 29-45.

[4] N New combinatorial interpretations of Ramanujan's partition congruences mod 5,7 and 11, Trans. Amer. Math. Soc. 305 (1988), no. 1, 47-77.

[5] _ A number-theoretic crank associated with open bosonic strings, Number Theory and Cryptography (Sydney, 1989) (J. H. Loxton, ed.), London Math. Soc. Lecture Note Ser., vol. 154, Cambridge University Press, Cambridge, 1990, pp. 221-226.

[6] G. H. Hardy and E. M. Wright, An Introduction to the Theory of Numbers, 3rd ed., Clarendon Press, Oxford University Press, London, 1954.

[7] S. Ramanujan, Congruence properties of partitions, Collected Papers of S. Ramanujan, Chelsea, New York, 1962, pp. 232-238.

Paul Hammond: Department of mathematics, University of Sussex, Falmer, Brighton BN1 9RF, UK

E-mail address: prhammond2001@yahoo.com

Richard Lewis: Department of Mathematics, The Open University, Walton Hall, Milton Keynes MK7 6AA, UK

E-mail address: rp146@tutor.open.ac.uk 


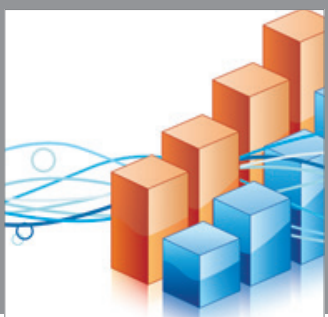

Advances in

Operations Research

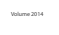

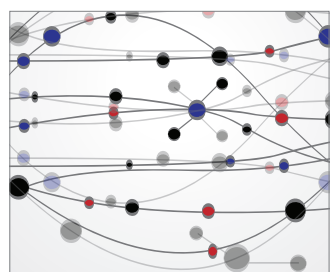

\section{The Scientific} World Journal
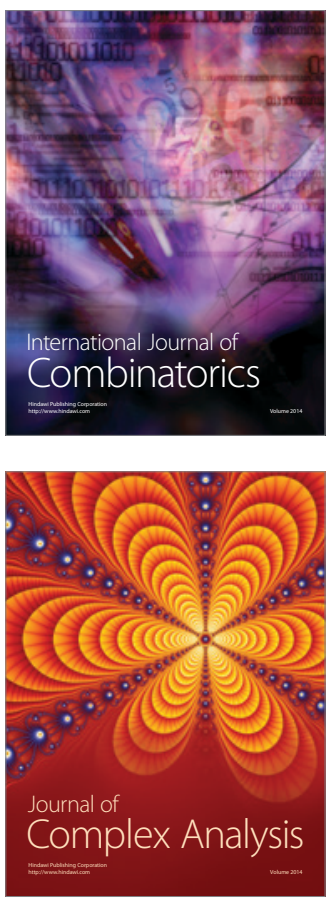

International Journal of

Mathematics and

Mathematical

Sciences
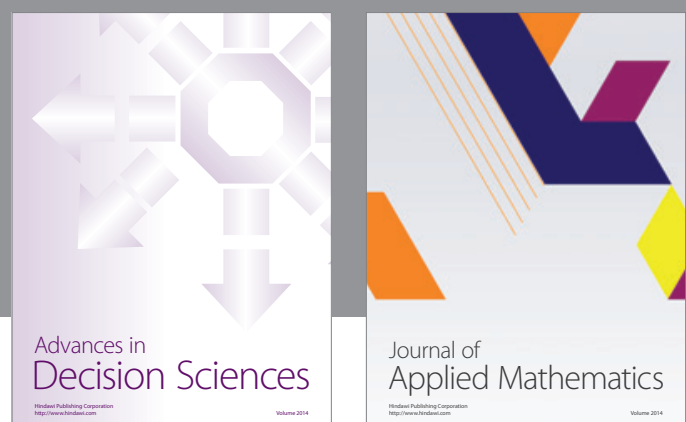

Journal of

Applied Mathematics
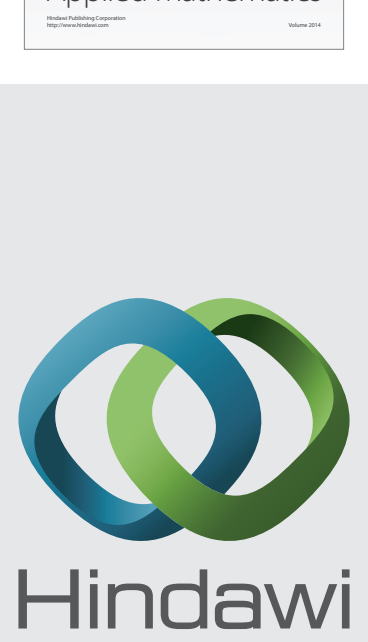

Submit your manuscripts at http://www.hindawi.com
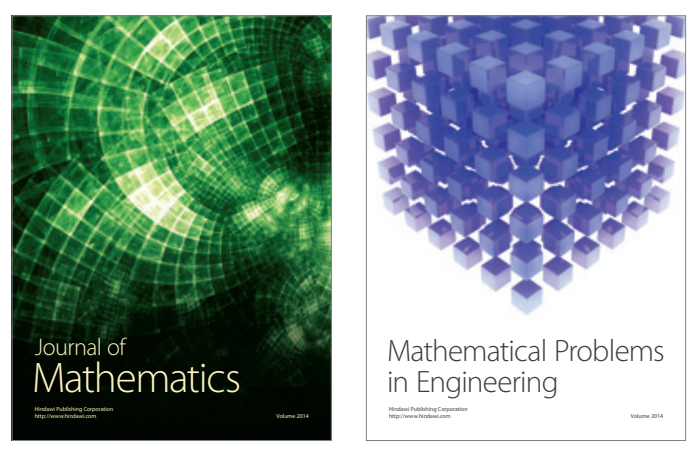

Mathematical Problems in Engineering
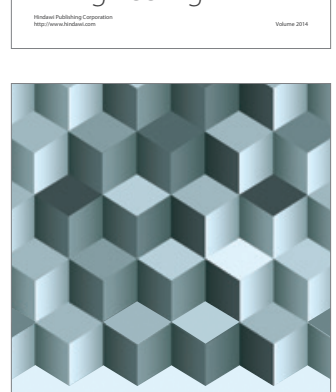

Journal of

Function Spaces
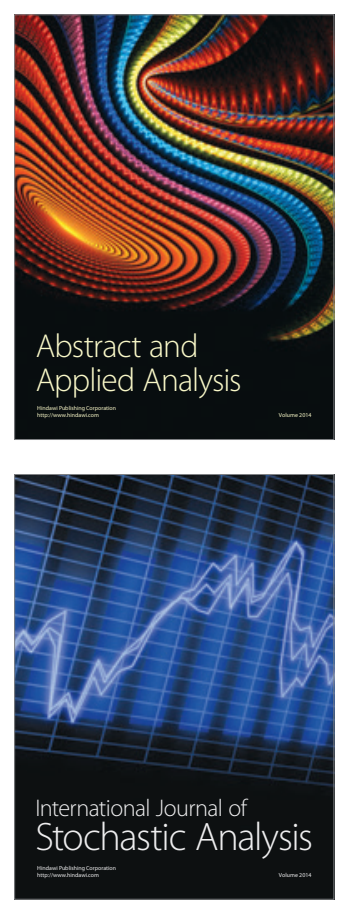

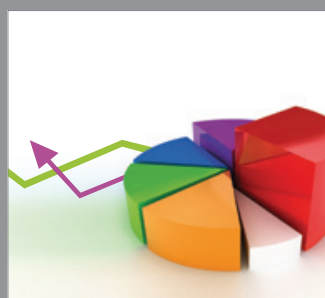

ournal of

Probability and Statistics

Promensencen
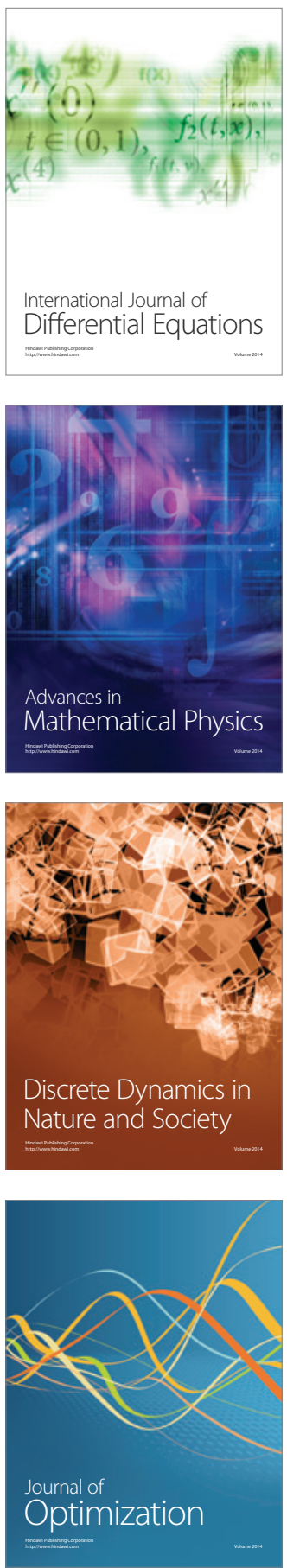